\title{
A fully photonics-based coherent radar system
}

\author{
Paolo Ghelfi ${ }^{1}$, Francesco Laghezza ${ }^{1}$, Filippo Scotti ${ }^{1}$, Giovanni Serafino ${ }^{2}$, Amerigo Capria ${ }^{3}$, Sergio Pinna ${ }^{2}$, Daniel Onori ${ }^{2}$, \\ Claudio Porzi ${ }^{2}$, Mirco Scaffardi ${ }^{1}$, Antonio Malacarne ${ }^{1}$, Valeria Vercesi ${ }^{2}$, Emma Lazzeri ${ }^{1}$, Fabrizio Berizzi ${ }^{3,4}$ \& Antonella Bogoni ${ }^{1}$
}

\begin{abstract}
The next generation of radar (radio detection and ranging) systems needs to be based on software-defined radio to adapt to variable environments, with higher carrier frequencies for smaller antennas and broadened bandwidth for increased resolution ${ }^{1-4}$. Today's digital microwave components (synthesizers and analogue-to-digital converters) suffer from limited bandwidth with high noise at increasing frequencies $^{5-7}$, so that fully digital radar systems can work up to only a few gigahertz, and noisy analogue up- and downconversions are necessary for higher frequencies. In contrast, photonics provide high precision and ultrawide bandwidth ${ }^{8,9}$, allowing both the flexible generation of extremely stable radio-frequency signals with arbitrary waveforms up to millimetre waves ${ }^{10-22}$, and the detection of such signals and their precise direct digitization without downconversion ${ }^{23-26}$. Until now, the photonics-based generation and detection of radiofrequency signals have been studied separately and have not been tested in a radar system. Here we present the development and the field trial results of a fully photonics-based coherent radar demonstrator carried out within the project PHODIR ${ }^{27}$. The proposed architecture exploits a single pulsed laser for generating tunable radar signals and receiving their echoes, avoiding radio-frequency up- and downconversion and guaranteeing both the software-defined approach and high resolution. Its performance exceeds state-of-the-art electronics at carrier frequencies above two gigahertz, and the detection of non-cooperating aeroplanes confirms the effectiveness and expected precision of the system.
\end{abstract}

The performance of the transceiver we developed is summarized in Table 1, together with a picture of the project demonstrator (Fig. 1). The transceiver enables radar systems with unprecedented frequency flexibility, signal quality and receiver resolution at high carrier frequency. The radar demonstrator has also been tested in a real environment.

The photonic generation of radio-frequency (RF) signals, avoiding microwave oscillators, can be realized either by means of opto-electronic oscillators $^{21,22}$ or by the heterodyning of two lasers in a photodiode ${ }^{10}$. The opto-electronic oscillators show excellent stability up to $40 \mathrm{GHz}$ with a miniaturized footprint, but their tunability is limited to a few gigahertz, whereas the heterodyning can generate any frequency up to the photodiode bandwidth, and is therefore more promising. Nevertheless, it requires the two lasers to be phase-locked. This can easily be obtained by selecting any pair of modes from the spectrum of modelocked lasers (MLLs) ${ }^{12,13}$, thanks to their intrinsic phase-locking condition. Moreover, selecting laser modes with variable wavelength detuning allows the flexible production of RF carriers with tunable frequency. Finally, the heterodyne method allows us to add an arbitrary modulation on the generated RF signal by modulating one of the beating laser modes.

The receivers for radar systems based on software-defined radio need analogue-to-digital converters (ADCs) that accept input signals over several tens of gigahertz, with fast sampling rates suitable for broadband signals and a high signal-to-noise ratio. Electronic ADCs are limited by the aperture jitter of the electronic sampling process-that is, the fluctuations of the interval duration between two consecutive sampleswhich spoils the accuracy of the digitized signal by adding a noise floor. Optical sampling can overcome this limitation ${ }^{23}$ : a train of short optical pulses can be directly modulated by the RF signal to be digitized, and then the peak amplitude of each optical pulse can be detected and digitized. Thus, sampling rates of several gigahertz are achievable, and the timing jitter of the sampling pulses can be much less than $100 \mathrm{fs}$. MLLs are particularly suitable pulse sources, with narrow pulsewidths and low temporal jitter.

The basic idea of the PHODIR project ('Photonics-based fully digital radar') is to use a single MLL for the realization of both the RF generation and sampling, obtaining an entire optics-based transceiver, as sketched in Fig. 2 (left), reducing the impact of photonics on the system costs. In the band-pass sampling process, which requires the sampling frequency to be at least twice the bandwidth of the pass-band signal, the carrier frequency of the sampled signal must not be a multiple of the sampling frequency. Unfortunately, this is the case when the same MLL is used for both generating and sampling the radar signal. This problem can be fixed by shifting one of the two MLL modes before heterodyning. The frequency shift must be driven by an RF oscillator with a negligible phase noise compared to the MLL, which is true if low-frequency oscillators

Table 1 | Performance of the photonics-based radar transceiver

\begin{tabular}{|c|c|c|}
\hline Parameter & Photonics-based transceiver & State-of-the-art electronics transceiver \\
\hline \multicolumn{3}{|c|}{ Transmitter } \\
\hline $\begin{array}{l}\text { Carrier frequency } \\
\text { Signal jitter } \\
\text { Signal-to-noise ratio } \\
\text { Spurious-free dynamic range } \\
\text { Instantaneous bandwidth }\end{array}$ & $\begin{array}{l}\text { Flexible direct generation up to } 40 \mathrm{GHz} \\
<15 \mathrm{fs} \text {; integrated from } 10 \mathrm{kHz} \text { to } 10 \mathrm{MHz} \\
>73 \mathrm{~dB} \mathrm{MHz} \\
>70 \mathrm{dBc} \\
200 \mathrm{MHz} \text {, easily extendable with } \mathrm{MLL} \text { at higher repetition rate }\end{array}$ & $\begin{array}{l}\text { Direct generation below } 2 \mathrm{GHz} \text {; up-conversions above } 2 \mathrm{GHz} \\
\text { Typical }>20 \mathrm{fs} \text {, integrated from } 10 \mathrm{kHz} \text { to } 10 \mathrm{MHz} \\
>80 \mathrm{~dB} \mathrm{MHz} \\
>70 \mathrm{dBc} \\
<2 \mathrm{GHz}\end{array}$ \\
\hline \multicolumn{3}{|c|}{ Receiver } \\
\hline $\begin{array}{l}\text { Input carrier frequency } \\
\text { Instantaneous bandwidth } \\
\text { Sampling jitter } \\
\text { Spurious-free dynamic range } \\
\text { Effective number of bits }\end{array}$ & $\begin{array}{l}\text { Up to } 40 \mathrm{GHz} \text {, with direct RF undersampling } \\
200 \mathrm{MHz} \text {, easily extendable with } \mathrm{MLL} \text { at higher repetition rate } \\
<10 \mathrm{fs} \text {, integrated from } 10 \mathrm{kHz} \text { to } 10 \mathrm{MHz} \\
50 \mathrm{~dB} \\
>7 \text { for carrier frequency up to } 40 \mathrm{GHz}\end{array}$ & $\begin{array}{l}<2 \mathrm{GHz} \text {; down-conversions at higher frequencies } \\
<2 \mathrm{GHz} \\
\text { Typical }>100 \mathrm{fs} \text {, integrated from } 10 \mathrm{kHz} \text { to } 10 \mathrm{MHz} \\
>70 \mathrm{~dB} \\
<8 \text { for carrier frequency }<2 \mathrm{GHz}\end{array}$ \\
\hline
\end{tabular}

Test results of the photonics-based transceiver are shown versus state-of-the-art electronic radar transceivers.

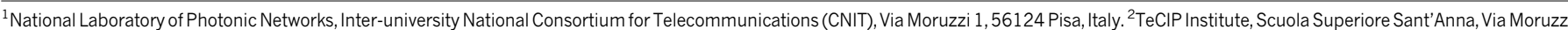

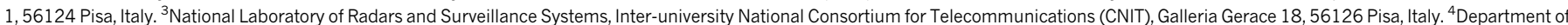
Information Engineering, University of Pisa, Via Caruso 16, 56122 Pisa, Italy. 


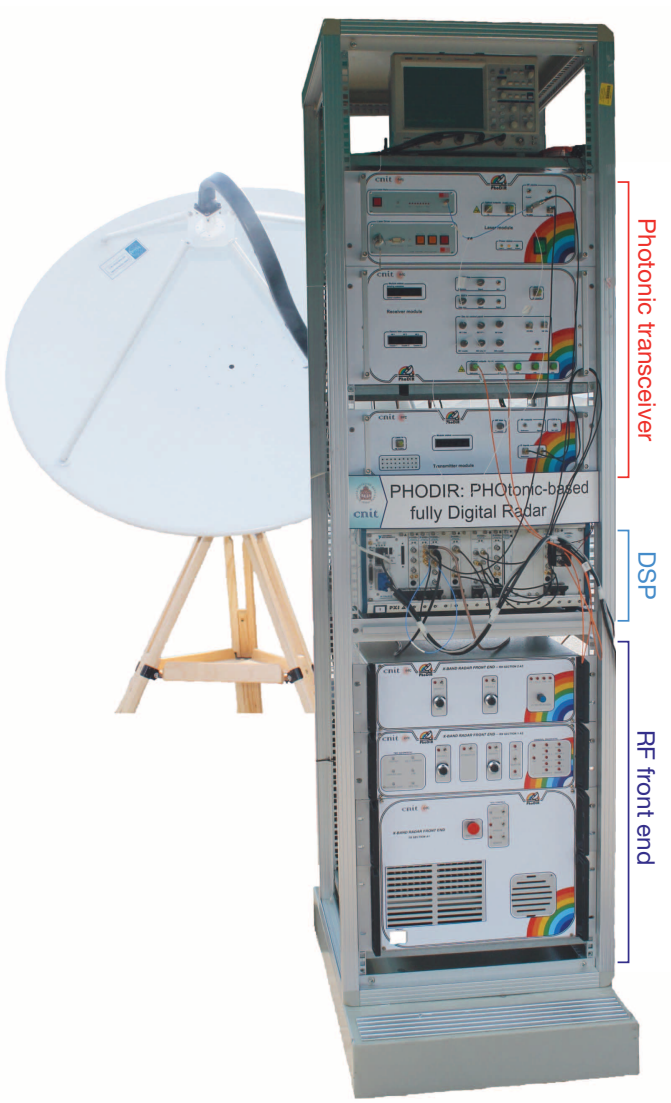

Figure $\mathbf{1} \mid$ The photonics-based radar transceiver. A picture (taken by A.B.) of the field-trial demonstrator. DSP, digital signal processor. are used ${ }^{28}$. Figure 2 (right) shows details of the PHODIR transceiver. In the photonics-based RF generator, two tunable optical filters select two modes from the spectrum of the MLL. One is modulated by the baseband radar pulse in a Mach-Zehnder modulator (MZM), whereas the other is shifted in frequency. In the photonics-based ADC, the optical pulses from the MLL are modulated in a MZM driven by the received echo signal. The modulated pulses are then parallelized in a serial-toparallel converter into multiple lower-rate sample streams so that, after opto-electronic conversion, multiple electronic ADCs with low sampling frequency and high precision can be used to digitize the pulse peak power carrying the sample information. The parallel digital samples are then interleaved in the digital signal processor to reconstruct the original signal. To match the pulse width to the photodiode bandwidth, spools of dispersion compensating fibre are inserted to broaden the optical pulses.

The precision of photonics is particularly valuable in coherent radar systems, which derive the speed of the targets from the Doppler frequency shift obtained by comparing the instantaneous frequency of the echoed signal with that of a continuous-wave reference signal. To allow this functionality, the PHODIR transceiver also generates the reference signal (see Fig. 2), exploiting a similar structure used for the radar pulse but without the modulation. Orthogonal sampling is implemented to detect the in-phase and quadrature components of the reference signal, allowing us to sample the reference signal only once and to derive the quadrature samples by shifting the in-phase samples ${ }^{1}$.

Figures 3 and 4 show the results obtained when testing the transceiver in the laboratory. The MLL we used generates subpicosecond pulses with a repetition rate of $400 \mathrm{MHz}$. The transmitter that we used differs slightly from the scheme in Fig. 2 in that the laser modes are not selected by tunable filters. Instead, the spectrum of the MLL is modulated by the radar pulse at an intermediate frequency equal to the frequency shift, and then photodetected. At the photodiode there are several pairs of modes that beat together at different frequencies, including the desired one. Given that all the beatings at the same frequency have the same phase, at the photodiode output a clear signal can be selected by a RF filter at the desired frequency. Therefore, here the tunability is moved

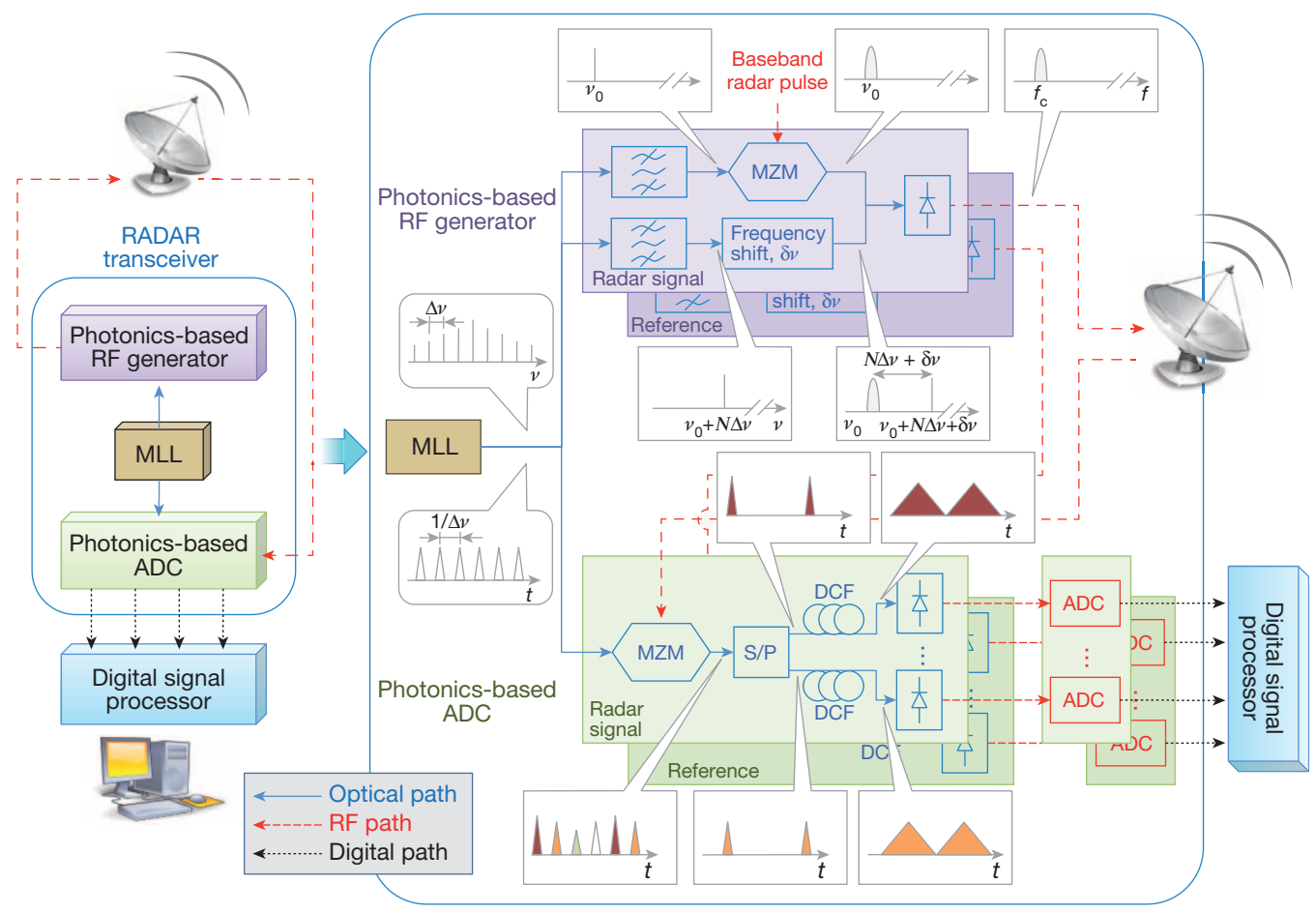

Figure $2 \mid$ Architecture of the photonics-based radar. Basic structure and detailed scheme of principle of the photonics-based coherent radar. S/P, series-to-parallel converter, DCF, dispersion compensating fibre. $t$, time; $v$, optical frequency; $\Delta v$, MLL repetition frequency; $\delta v$, applied frequency shift; $f$, RF frequency; $N$, positive integer. 

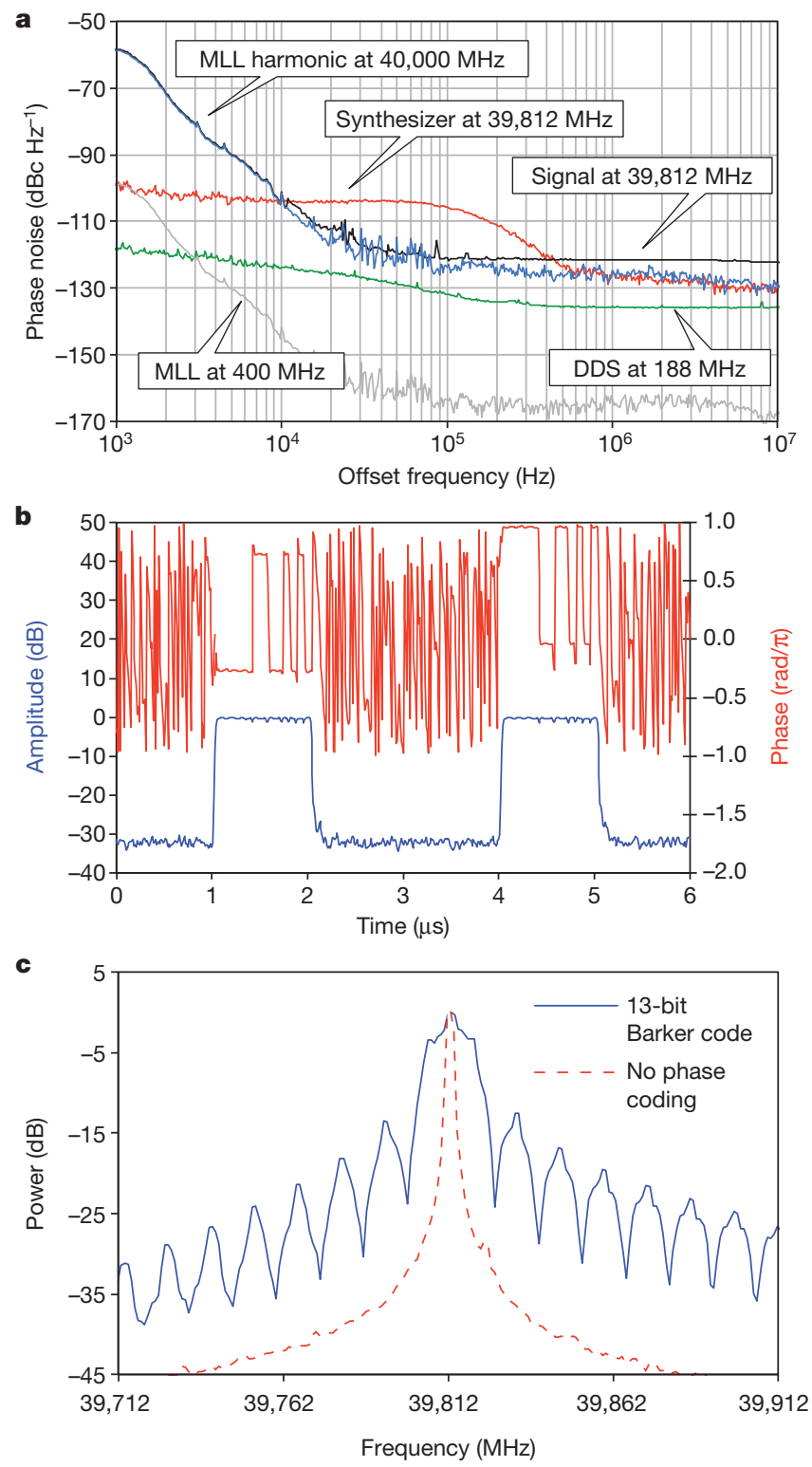

Figure 3 Test results of the photonics-based RF generator. a, Phase noise curve of the RF signal optically generated at $39,812 \mathrm{MHz}$, compared with the phase noise curves of the fundamental frequency of the laser at $400 \mathrm{MHz}$, of its hundredth multiple at $40,000 \mathrm{MHz}$, of the continuous-wave intermediate frequency at $188 \mathrm{MHz}$ generated by a direct digital synthesizer, and of a signal at $39,812 \mathrm{MHz}$ generated by a synthesizer. $\mathbf{b}$, Amplitude and phase transients of a $1-\mu$ s radar pulse phase-modulated by a 13 -bit Barker code with carrier frequency of 39,812 MHz.c, RF spectrum of the 13-bit Barker coded radar pulse at $39,812 \mathrm{MHz}$, compared with the RF spectrum of the uncoded pulse.

in the RF domain by means of selectable RF filters. In any case, this does not affect the photonics-based approach because the stability and flexibility of the generated RF signals are only obtainable using photonics.

We tested the photonics-based RF generator, producing signals with carriers up to $40 \mathrm{GHz}$ (limited by the photodiode bandwidth). As an example, Fig. 3 reports the results obtained at 39,812 MHz. Figure 3a reports the stability, in terms of the phase noise curve, of the optically generated RF carrier, which is obtained applying a shift of $188 \mathrm{MHz}$ to the hundredth multiple of the MLL fundamental frequency. The graph also reports the phase noise of the detected fundamental frequency of the laser at $400 \mathrm{MHz}$, of its hundredth multiple at $40,000 \mathrm{MHz}$, and of the continuous-wave intermediate frequency at $188 \mathrm{MHz}$ generated by a direct digital synthesizer. As expected ${ }^{12}$, the phase noise curve of the $40,000 \mathrm{MHz}$ signal is equivalent to the fundamental phase noise curve
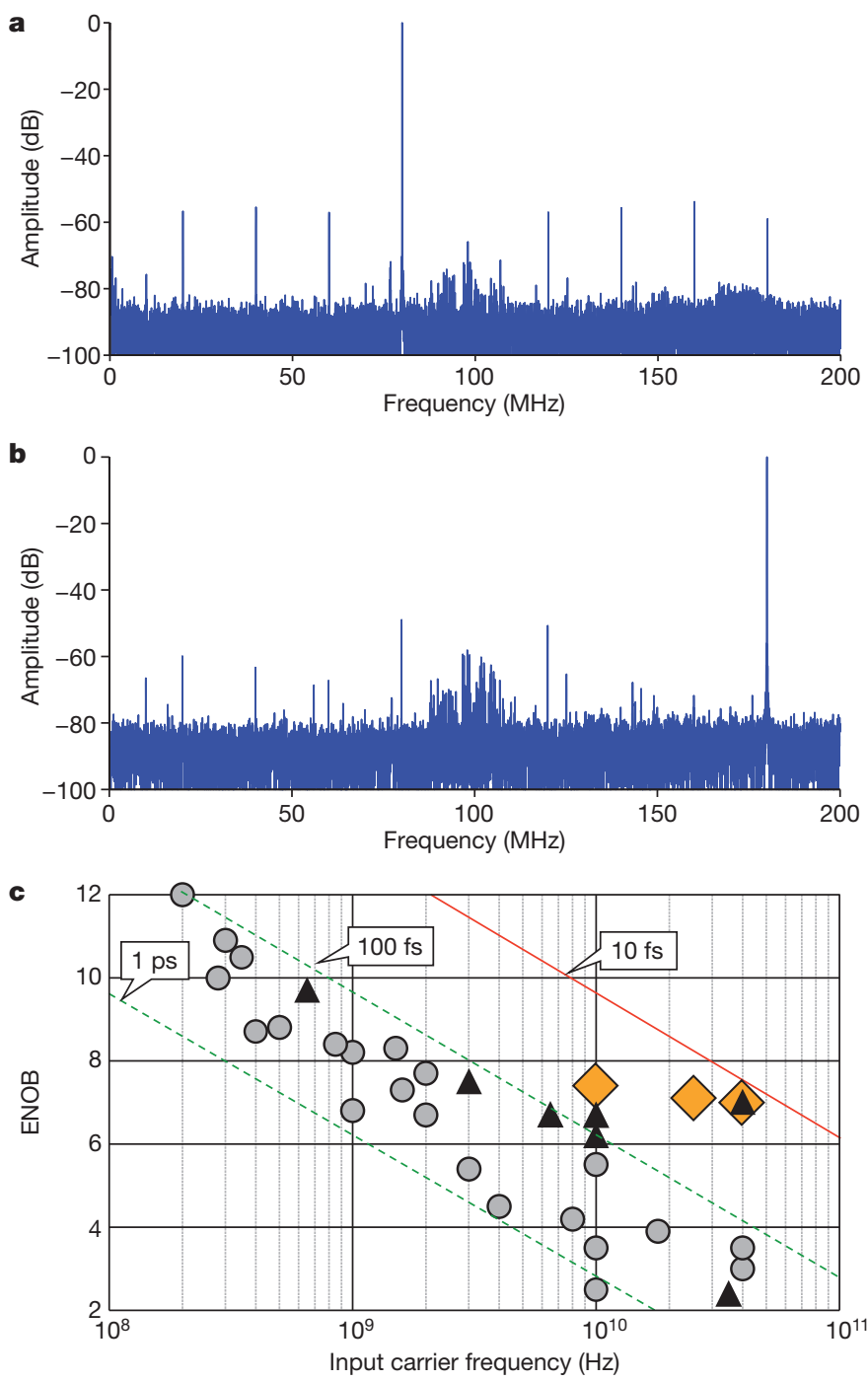

$\diamond$ This work $\triangle$ Photonic ADCs OElectronic ADCs

Figure $4 \mid$ Test results of the photonics-based ADC. a, b, Fast Fourier transform spectrum of the RF signal at $9,920 \mathrm{MHz}(\mathbf{a})$ and $39,820 \mathrm{MHz}(\mathbf{b})$, optically sampled at $400 \times 10^{6}$ samples per second and digitized at

$4 \times 100 \times 10^{6}$ samples per second. c, Extended Walden plot, comparing the performance of electronic and photonic ADCs.

of the laser shifted up by $40 \mathrm{~dB}$. The signal at intermediate frequency shows significantly lower phase noise than that at $40,000 \mathrm{MHz}$, so the signal at $39,812 \mathrm{MHz}$ substantially maintains the noise characteristics of the non-shifted signal. The higher noise floor at high offset frequency is induced by the necessary RF amplifiers, and does not substantially affect the signal stability. Given that the typical radar pulse repetition time is about $100 \mu \mathrm{s}$, the phase noise curve is of interest for offset frequencies from $10 \mathrm{kHz}$ and up. The timing jitter can be calculated by integrating the phase noise over this range, which turns out to be $10 \mathrm{fs}$ for the fundamental $400 \mathrm{MHz}$ and its multiple at $40,000 \mathrm{MHz}$, and $15 \mathrm{fs}$ for the shifted signal. For comparison, Fig. 3a also reports the phase noise curve of a signal at 39,812 MHz generated by a state-of-the-art synthesizer, which shows a jitter of $23 \mathrm{fs}$. Our scheme therefore performs significantly better than the synthesizer. A spectrum analysis of the photonics-generated carrier also shows a signal-to-noise ratio higher than $73 \mathrm{~dB}$ on a noise bandwidth of $1 \mathrm{MHz}$, and a spurious-free dynamic range of $>70 \mathrm{dBc}$ (decibels relative to the carrier power). Analogous results have been obtained generating signals in the range from a few hertz to $40 \mathrm{GHz}$ by substituting the RF filter after the photodiode with others at a different central frequency, and setting the direct digital 
synthesizer to generate a different intermediate frequency. This demonstrates that the proposed method can effectively generate very stable RF signals at arbitrary frequencies over an unprecedented broad range. Performance improvements can be obtained by exploiting photonic integration techniques.

By setting the direct digital synthesizer to generate modulated signals at intermediate frequency rather than continuous-wave signals, we can move the modulation signals on the desired RF carrier. Radar pulses can therefore be directly produced on the RF carrier by digitally generating pulse-modulated signals at intermediate frequency. Moreover, by adding a phase modulation to the digital signal from the synthesizer, phase-coded radar pulses can be realized for increasing the radar resolution through spectral broadening. Figure $3 \mathrm{~b}$ reports the amplitude and phase transients of the RF signal at $39,812 \mathrm{MHz}$ that are produced when the intermediate frequency is modulated by $1-\mu$ s rectangular pulses with the typical 13-bit Barker code phase modulation. Figure $3 \mathrm{c}$ clearly shows the expected pulse spectrum broadening from $1 \mathrm{MHz}$ to $13 \mathrm{MHz}$, induced by the phase modulation. The bandwidth could be extended up to half the repetition rate of the MLL $(200 \mathrm{MHz})$. These results confirm the capability of the scheme to generate arbitrary amplitude-modulated and/or phase-modulated signals ${ }^{19}$.

Figure 4 reports the results obtained by testing the photonics-based ADC of the PHODIR system ${ }^{26}$. The RF signal is optically sampled at $400 \times 10^{6}$ samples per second in a MZM with $40-\mathrm{GHz}$ bandwidth. The modulated optical pulses are parallelized into four streams at $100 \times 10^{6}$ samples per second, exploiting an integrated $1 \times 4 \mathrm{LiNbO}_{3}$ switching matrix, ensuring an extinction ratio-that is, a power ratio between a selected output port and the three disabled ones-of $>25 \mathrm{~dB}$ (ref. 29). The pulses are broadened in four spools of dispersion compensating fibre and digitized by four electronic ADCs synchronized with the pulse peaks. Finally, a digital signal processor interleaves the samples. The photonics-based ADC is tested with input continuous-wave signals up to $40 \mathrm{GHz}$. As examples, Fig. $4 \mathrm{a}$ and b report the calculated fast Fourier transform for signals at 9,920 MHz and $39,820 \mathrm{MHz}$, both with $4 \times 10,000$ points, after the digital processing. The undersampling process aliases the signals in the Nyquist bandwidth (direct current to $200 \mathrm{MHz}$ ), at $80 \mathrm{MHz}$ and $180 \mathrm{MHz}$, respectively. The noise floor in the two graphs appears at different levels owing to the increasing influence of the sampling jitter for increasing signal frequency. The residual spurious tones after the data post-processing are several tens of decibels below the signal, enabling a spurious-free dynamic range of $>50 \mathrm{~dB}$. A higher spurious-free dynamic range could be obtained with a more precise sample parallelization process. By integrating the fast Fourier transform it is possible to calculate the signal-to-noise and distortion ratios, and from this the effective number of bits. In Fig. 4c the so-called 'Walden plot' is shown, reporting the performance comparison in terms of input carrier frequency and the effective number of bits of the best electrical and photonic ADCs reported so $\mathrm{far}^{7}$, and of the PHODIR receiver. The theoretical upper bounds caused by different sampling jitters are also reported. The jitter of the MLL obtained by integrating its phase noise curve from $10 \mathrm{kHz}$ is about $10 \mathrm{fs}$, whereas the best electronic ADCs show an aperture jitter of about $100 \mathrm{fs}$. As a consequence, our system reaches an effective number of bits of 7.4 for an input signal at 9,920 MHz, and 7 at $39,820 \mathrm{MHz}$, performing significantly better than the reported electrical ADCs. At $40 \mathrm{GHz}$, the system performance is close to the theoretical upper bound for $10 \mathrm{fs}$ of aperture jitter, but it moves away from the upper bound at lower signal frequencies, indicating that at lower frequency the system's effective number of bits is limited by the spurious tones, whereas at higher frequency it is mostly limited by the time jitter of the MLL, because the spurious tones become comparable with the noise floor. Improvements in the effective number of bits could be achieved by including a differential detection scheme to suppress the detected noise ${ }^{24}$, and an improved switching matrix with higher extinction to reduce the power of the spurious tones. Moreover, a larger instantaneous bandwidth could be achieved with higher sampling rates.

The test results of the PHODIR transceiver are summarized in Table 1, and compared with the performance of state-of-the-art electronic radar transceivers ${ }^{6}$. The advantages of the photonic approach are evident in the extreme frequency flexibility over tens of gigahertz, in the arbitrary modulation capability, and in the precision of the digitization for any input frequency. We believe that these features will enable the use of software-defined radio in future radar systems.

We also tested the PHODIR transceiver reported above in a fieldtrial demonstrator to determine its effectiveness in a radar system. For this purpose, we used a RF front-end (RF circulator, switches, amplifiers, filters and bistatic antenna) for a signal carrier at 9,900 MHz. Noncooperating (that is, normally operating and unaware of our detection) aeroplanes were detected as dynamic targets. For example, Fig. 5a reports the trajectory of an observed aeroplane during takeoff. The range-velocity map of the target at point A of the trajectory is reported in Fig. 5b. The velocity and position of the target were cross-checked by trajectory data provided by public websites (http://www.flightradar24.com/). At the detection point, the known radial velocity of the target is about $96 \mathrm{~km} \mathrm{~h}^{-1}$ (for a real speed of about $400 \mathrm{~km} \mathrm{~h}^{-1}$ ), and the known distance is about $5.5 \mathrm{~km}$. The corresponding detected signal shows the target at $5.4 \mathrm{~km}$ with a radial velocity of $95 \mathrm{~km} \mathrm{~h}^{-1}$. Figure $5 \mathrm{c}$ shows a a

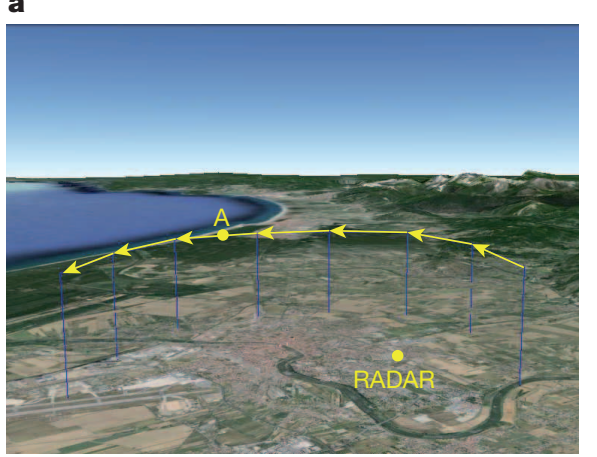

b
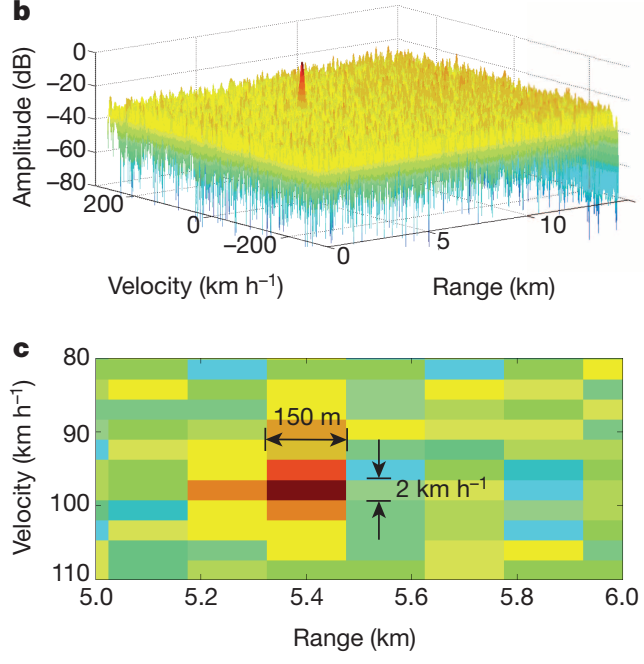

d

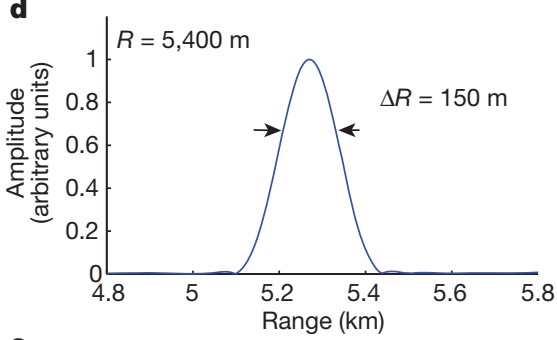

e

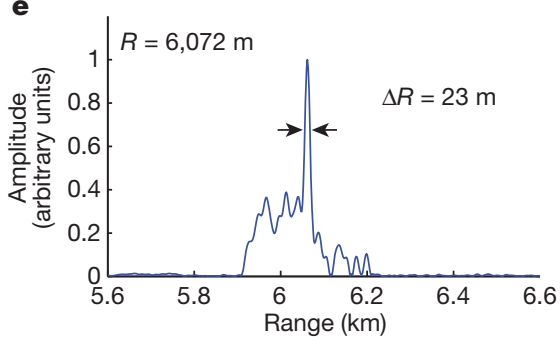

Figure $5 \mid$ Test results of the field-trial demonstrator. a, Trajectory of a target aeroplane. b, Range-velocity map at point A in the trajectory. c, Zoom showing the range and velocity resolution. $\mathbf{d}, \mathbf{e}$, Range profile without (d) or with (e) pulse coding. 
two-dimensional zoom of the range-velocity map, with distance resolution of $150 \mathrm{~m}$ and velocity resolution of $2 \mathrm{~km} \mathrm{~h}^{-1}$. The target shows a detected echo $45 \mathrm{~dB}$ above the background. Finally, a 13-bit Barker code was modulated on the phase of the radar pulses to increase the resolution. Figure $5 \mathrm{~d}$ and e report the range profile collected with and without this pulse coding, showing an increase in precision from $150 \mathrm{~m}$ to $23 \mathrm{~m}$.

The PHODIR project has pioneered a fully photonics-based radar system, enabling the software-defined radio approach with a performance exceeding that of state-of-the-art radar systems. In particular, today's electronic transceivers cannot achieve the same frequency range without the use of several parallel architectures, and do not provide an equivalent precision, especially at high carrier frequencies. A field-trial demonstrator has proved the effectiveness and the expected precision of the photonic architecture. This proof-of-concept system has been realized using commercial devices, except for the switching matrix which was developed within the EU programme NEXPRESSO ${ }^{29}$. We are now planning to develop dedicated photonic integrated circuits, which could fully realize the potential of the photonic approach, leading to compact, flexible systems with high performance for the most advanced applications, such as simultaneous surveillance and communications. The use of photonics in radar systems also allow additional functionalities, which make its use even more convenient. For example, tunable time delay can be applied to the lasers transporting the RF signals to control the beamforming at arrayed antennas ${ }^{30}$. Moreover, photonics permits the use of optical fibres for transporting the radar signals to and from the antenna, allowing its remotization with negligible losses. Therefore, the architecture we propose, as sketched in Fig. 2, should open up new frontiers in the field of radar, enabling future smart multifunction surveillance systems.

\section{METHODS SUMMARY}

We use the OneFive Origami passive MLL with a pulse repetition frequency of $400 \mathrm{MHz}$ and an optical bandwidth of about $8 \mathrm{~nm}$ at a wavelength of $1,560 \mathrm{~nm}$. In photonics-based signal generation, the frequency shift of one of the modes of the MLL can be realized, for example, by single-sideband modulation with carrier suppression. The state-of-the-art synthesizer used as a reference in the analysis of the phase noise of the photonics-generated signal is the Agilent E8257D. The integrated $1 \times 4 \mathrm{LiNbO}_{3}$ switching matrix employed at the receiver stage consists of three dual-output MZMs in a cascaded configuration. The chromatic dispersion of the spools of dispersion compensating fibre before the photodiodes is $-300 \mathrm{ps} \mathrm{nm}^{-1}$. The electronic ADCs are the data acquisition boards NI PXIe5122 from National Instruments, with 14 -bit resolution and $100 \times 10^{6}$ samples per second. The digital processing implemented to interleave the samples also suppresses the spurious tones caused by the nonlinearity of the optical modulator and the different attenuations and delays of the four parallel branches of the photonics-based receiver. The synthesizer used for testing the photonic ADC is the Agilent E8257D. In the RF front-end of the PHODIR demonstrator, the RF filter at the receiver has a bandwidth of $40 \mathrm{MHz}$. Therefore both the received echo and the reference signal are optically sampled at $400 \times 10^{6}$ samples per second, and digitized by acquiring their aliased replica with ADCs at $80 \times 10^{6}$ samples per second. The non-modulated 1 - $\mu$ s-long radar pulses give a range resolution of $150 \mathrm{~m}$, with a velocity resolution of $2 \mathrm{~km} \mathrm{~h}^{-1}$ given by the coherent integration time of $20 \mathrm{~ms}$. In the field trial, we ran a preliminary system calibration focusing on static targets, detecting objects in a range up to $30 \mathrm{~km}$ with a transmitted RF peak power of about $20 \mathrm{~W}$.

\section{Received 14 October 2013; accepted 23 January 2014.}

1. Skolnik, M. L. Introduction to Radar Systems 3rd edn (McGraw-Hill, 1980).

2. Haykin, S. Cognitive radar: a way of the future. IEEE Signal Process. Mag. 23, 30-40 (2006).

3. Ravenni, V. Performance evaluations of frequency diversity radar system. Proc. Eur. Microwave Conf. 1715-1718, http://dx.doi.org/10.1109/eumc.2007.4405545 (2007).

4. Tsui, J. B. Digital Techniques for Wideband Receivers 2nd edn (SciTech, 2004).

5. Scheer, J. A. \& Kurtz, J. L. Coherent Radar Performance Estimation (Artech House, 1993).

6. Richards, M. A. Scheer, J. A. \& Holm, W. A. Principle of Modern Radar: Basic Principles (SciTech Publishing, 2010).
7. Walden, R. Analog-to-Digital Conversion in the Early Twenty-First Century (Wiley Encyclopedia of Computer Science and Engineering, 2008).

8. Capmany, J. \& Novak, D. Microwave photonics combines two worlds. Nature Photon. 1, 319-330 (2007)

9. Yao, J. Microwave photonics. J. Lightwave Technol. 27, 314-335 (2009)

10. Goldberg, L., Esman, R. D. \& Williams, K. J. Generation and control of microwave signals by optical techniques. IEEE Proc. J. 139, 288-295 (1992)

11. Khan, M. H. et al. Ultrabroad-bandwidth arbitrary radiofrequency waveform generation with a silicon photonic chip-based spectral shaper. Nature Photon. $\mathbf{4}$ 117-122 (2010).

12. Serafino, G. et al. Stable optically generated RF signals from a fibre mode-locked laser. Proc. 23rd IEEE Photonics Soc. Ann. Meet. Abstr. TuK4, 193-194, http:// dx.doi.org/10.1109/photonics.2010.5698824 (2010).

13. Yilmaz, T., DePriest, C. M., Turpin, T., Abeles, J. H. \& Delfyett, P. J. Toward a photonic arbitrary waveform generator using a modelocked external cavity semiconductor laser. IEEE Photonics Technol. Lett. 14, 1608-1610 (2002).

14. Chou, J., Han, Y. \& Jalali, B. Adaptive RF-photonic arbitrary waveform generator. IEEE Photonics Technol. Lett. 15, 581-583 (2003).

15. McKinney, J. D., Leaird, D. E. \& Weiner, A. M. Millimeter-wave arbitrary waveform generation with a direct space-to-time pulse shaper. Opt. Lett. 27, 1345-1347 (2002).

16. Lin, I. S., McKinney, J. D. \& Weiner, A. M. Photonic synthesis of broadband microwave arbitrary waveform applicable to ultrawideband communication. IEEE Microwave Wireless Components Lett. 15, 226-228 (2005).

17. Chi, H. \& Yao, J. P. An approach to photonic generation of high frequency phase-coded RF pulses. IEEE Photonics Technol. Lett. 19, 768-770 (2007).

18. Li, Z., Li, W., Chi, H., Zhang, X. \& Yao, J. Photonic generation of phase-coded microwave signal with large frequency tunability. IEEE Photonics Technol. Lett. $\mathbf{2 3}$ 712-714 (2011).

19. Ghelfi, P., Scotti, F., Laghezza, F. \& Bogoni, A. Phase coding of RF pulses in photonics-aided frequency-agile coherent radar systems. IEEE J. Quantum Electron. 48, 1151-1157 (2012).

20. Ghelfi, P., Scotti, F., Laghezza, F. \& Bogoni, A. Photonic generation of phase-modulated RF signals for pulse compression techniques in coherent radars. J. Lightwave Technol. 30, 1638-1644 (2012).

21. Maleki, L. et al. High performance, miniature hyper-parametric microwave photonic oscillator. Proc. IEEE Freq. Control Symp. 558-563, http://dx.doi.org/ 10.1109/freq.2010.5556265 (2010).

22. Maleki, L. The optoelectronic oscillator. Nature Photon. 5, 728-730 (2011).

23. Valley, G. C. Photonic analog-to-digital converters. Opt. Exp. 15, 1955-1982 (2007).

24. Khilo, A. etal. Photonic ADC: overcoming the bottleneck of electronic jitter. Opt. Exp. 20, 4454-4469 (2012)

25. Chou, J., Conway, J. A., Sefler, G. A., Valley, G. C. \& Jalali, B. Photonic bandwidth compression front end for digital oscilloscopes. J. Lightwave Technol. 27, 5073-5077 (2009)

26. Laghezza, F. et al. Jitter-limited photonic analog-to-digital converter with 7 effective bits for wideband radar applications. Proc. IEEE Radar Conf. 1-5, http://dx.doi.org/10.1109/radar.2013.6586075 (2013)

27. Photonic-based Fully Digital Radar (PHODIR) http://www.phodir.eu (2009)

28. Ghelfi, P., Scotti, F., Nguyen, A. T., Serafino, G. \& Bogoni, A. Novel architecture for a photonics-assisted radar transceiver based on a single mode-locking laser. IEEE Photonics Technol. Lett. 23, 639-641 (2011).

29. Pierno, L. et al. Optical switching matrix as time domain demultiplexer in photonic ADC. Proc. Eur. Microwave Integr. Circuits Conf. Abstr. EuMIC03.3, INSPEC accession number 13990947 (2013).

30. Ghelfi, P. et al. Photonic generation and independent steering of multiple RF signals for software defined radars. Opt. Exp. 21, 22905-22910 (2013)

Acknowledgements This work was supported by the ERC projects PHODIR (contract number 239640) and PREPARE (contract number 324629), and by the EU NEXPRESSO programme through the project INSIDE with Selex Sistemi Integrati S.p.A. (now Selex ES S.p.A.)

Author Contributions A.B. coordinated all the activities of the PHODIR project. A.B. and P.G. designed the architecture of the photonics-based transceiver and wrote the paper. F.L., A.C. and F.B. defined the radar parameters and designed the RF front end. F.L., F.S. S.P., G.S., E.L. and D.O. implemented the photonic subsystems. S.P. designed and developed the electronic controls of the machine that separates the samples into parallel streams and of the front panels of the radar demonstrator. F.S. assembled the demonstrator. F.L. implemented the digital processing tools. P.G. G.S., M.S., E.L. and A.B. analysed and discussed the results from the photonics-based transmitter.P.G., F.L., F.S., S.P., D.O., A.M. and A.B. analysed and discussed the results from the photonics-based receiver. F.L.F.S. S.P. G.S. and D.O. collected and processed the data of the field trial. P.G., F.L., F.S., G.S., S.P., D.O. and A.B. analysed and discussed the results of the field trial. C.P., V.V., P.G. and A.B. discussed the possible development of the photonics-based transceiver with integrated photonics techniques. P.G., G.S., F.L., F.S. and A.B. commented on the manuscript.

Author Information Reprints and permissions information is available at www.nature.com/reprints. The authors declare no competing financial interests. Readers are welcome to comment on the online version of the paper. Correspondence and requests for materials should be addressed to A.B. (antonella.bogoni@cnit.it). 